\title{
On the $L^{\infty}$-Convergence of Galerkin Approximations for Second-Order Hyperbolic Equations
}

\author{
By Garth A. Baker* and Vassilios A. Dougalis**
}

\begin{abstract}
It is shown that certain classes of high order accurate Galerkin approximations for homogeneous second-order hyperbolic equations, known to possess optimal order rate of convergence in $L^{2}$, also possess optimal order rate of convergence in $L^{\infty}$.

This is attainable with particular smoothness assumptions on the initial data. We establish sufficient conditions for optimal $L^{\infty}$-convergence of the approximations to the solution and also the approximation to its time derivative. This is done for both semidiscrete approximations and for single-step fully discrete approximations generated by rational functions.
\end{abstract}

1. Introduction. We consider approximating the solution of the following initialboundary value problem. $\Omega$ will be a bounded domain in $\mathbf{R}^{N}$ with boundary $\partial \Omega$ assumed to be an $(N-1)$-dimensional manifold of class $C^{\infty}$. For a fixed $0<t^{*}<\infty$, a real-valued function $u$ is sought satisfying

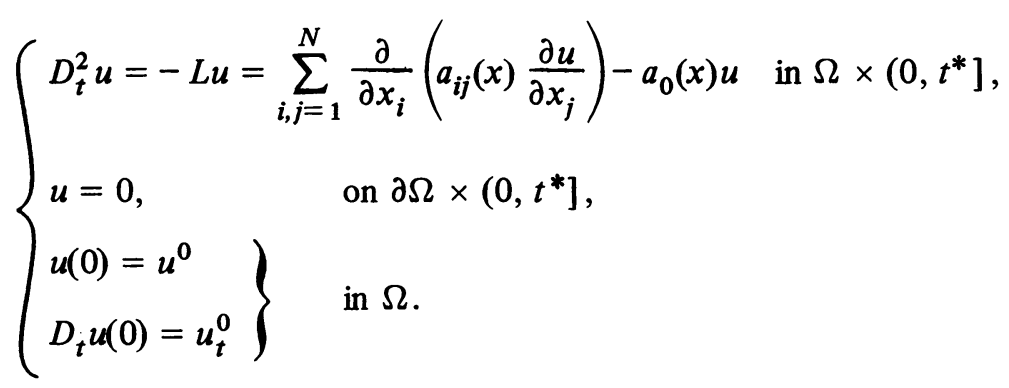

$u^{0}, u_{t}^{0}$ are given functions,

$$
a_{i j}=a_{j i} \in C^{\infty}(\bar{\Omega}), \quad i, j=1,2, \ldots, N,
$$

and $a_{0} \in C^{\infty}(\bar{\Omega})$ with $a_{0} \geqslant 0$ in $\bar{\Omega}$. $L$ will be assumed to be uniformly elliptic, i.e., for some constant $\underline{\alpha}>0$,

$$
\sum_{i, j=1}^{N} a_{i j}(x) \xi_{i} \xi_{j} \geqslant \underline{\alpha} \sum_{i=1}^{N} \xi_{i}^{2},
$$

for all $x \in \bar{\Omega}$ and all $\left(\xi_{1}, \ldots, \xi_{N}\right) \in \mathbf{R}^{N}$.

Semidiscrete and fully discrete Galerkin approximations in the context of existing finite element methods have been considered by Dupont [8], Baker [1], Crouzeix

Received August 23, 1978; revised July 5, 1979.

AMS (MOS) subject classifications (1970). Primary 65M15, 65N30.

* Supported by ONR Grant N00014-57-A-0298-0015.

** Supported by USARO Grant DAAG29-78-C-0024. 
[7], and Baker and Bramble [2], among others. In these works optimal order rate of convergence estimates in $L^{2}(\Omega)$ have been obtained.

In this paper we are concerned with the convergence in $L^{\infty}(\Omega)$ of such approximation schemes. Our approach is motivated by techniques of $L^{\infty}(\Omega)$-estimation initiated for parabolic equations in [4], [3], adopting the first-order system formulation for second-order hyperbolic equations of [1], [2], [7] .

In the special case of one space dimension, optimal $L^{\infty}$-estimates have been obtained by Wheeler [16] and Wahlbin [15]. Specifically, the estimates are derived for both the semidiscrete approximation and for a particular second-order accurate in time, two-step, fully discrete scheme devised in [8], the techniques used being clearly restricted to one space dimension.

In this work we attack the general problem (1.1). Our techniques yield for the semidiscrete approximation optimal $L^{\infty}(\Omega)$-convergence for the error, as well as for the time derivative of the error, under suitable smoothness assumptions on $u^{0}$ and $u_{t}^{0}$. Of prime interest is a class of fully discrete schemes generated by rational functions, devised in [2], [7]. For these high order accurate in time schemes we obtain optimal $L^{\infty}(\Omega)$-convergence of the approximation to the solution, and also for the approximation to its time derivative, occurring naturally from the first-order formulation.

The remainder of this section is devoted to establishing the notation and a precise statement of the main results.

For $S \geqslant 0, H^{S}(\Omega)$ will denote the Sobolev space $W_{2}^{S}(\Omega)$ of real-valued functions on $\Omega$, the norm on which we denote by $\|\cdot\|_{H} s_{(\Omega)}$. The inner product on $L^{2}(\Omega)=$ $H^{0}(\Omega)$ we denote by $(\cdot, \cdot)$ and the associated norm by $\|\cdot\|$. The norm on $L^{\infty}(\Omega)$ we denote by $|\cdot|$ and, in general, that on the Sobolev space $W_{\infty}^{m}(\Omega)$ by $|\cdot|_{m}, m \geqslant 0$. $\left\{\lambda_{j}\right\}_{j \geqslant 1}$ will denote the eigenvalues, in nondecreasing order, of the elliptic operator $L$ on $\Omega$; the corresponding set of eigenfunctions (with boundary values zero) is $\left\{\varphi_{j}\right\}_{j \geqslant 1}$ $\subset C^{\infty}(\Omega)$. This set is assumed to be orthonormal in $L^{2}(\Omega)$. For real $S$, we define the space

$$
\dot{H}^{S}(\Omega)=\left\{v \in L^{2}(\Omega):\|v\|_{S}=\left(\sum_{j} \lambda_{j}^{S}\left|\left(v, \varphi_{j}\right)\right|^{2}\right)^{1 / 2}<\infty\right\} .
$$

Following [4], it is easily seen that for integer $S \geqslant 0$,

$$
\dot{H}^{S}(\Omega)=\left\{v \in H^{S}(\Omega): L^{j} v=0 \text { on } \partial \Omega, \text { for } j<S / 2\right\},
$$

and the norms $\|\cdot\|_{H} s_{(\Omega)}$ and $\|\cdot\|_{S}$ are equivalent on $\dot{H}^{S}(\Omega)$. The solution of (1.1) is given by

$$
u(t)=\sum_{j}\left[\cos \lambda_{j}^{1 / 2} t\left(u^{0}, \varphi_{j}\right)+\lambda_{j}^{-1 / 2} \sin \lambda_{j}^{1 / 2} t\left(u_{t}^{0}, \varphi_{j}\right)\right] \varphi_{j}
$$

from which we obtain the conservation law

$$
\|u(t)\|_{S}^{2}+\left\|D_{t} u(t)\right\|_{S-1}^{2}=\left\|u^{0}\right\|_{S}^{2}+\left\|u_{t}^{0}\right\|_{S-1}^{2},
$$

for $t \geqslant 0$ and $S \geqslant 0$. 
In order to exploit the property (1.2) we introduce the generalized "energy" spaces $\dot{H}^{S}=\dot{H}^{S}(\Omega) \times \dot{H}^{S-1}(\Omega)$, for $S \geqslant 1$, furnished with the norms $\|\cdot\| \cdot \|_{S}$, defined as follows. A generic point of $\dot{H}^{S}$ we denote by

$$
V=\left(\begin{array}{l}
v_{1} \\
v_{2}
\end{array}\right)
$$

and define

$$
\|V\|_{S}=\left(\left\|v_{1}\right\|_{S}^{2}+\left\|v_{2}\right\|_{S-1}^{2}\right)^{1 / 2}, \quad S \geqslant 1 .
$$

Let $a(\cdot, \cdot)$ denote the form

$$
a(\varphi, \psi)=\int_{\Omega}\left\{\sum_{i, j=1}^{N} a_{i j} \frac{\partial \varphi}{\partial x_{j}} \frac{\partial \psi}{\partial x_{i}}+a_{0} \varphi \psi\right\} d x, \quad \varphi, \psi \in H^{1}(\Omega) .
$$

We shall work with the solution operator $T: H^{S}(\Omega) \rightarrow H^{S+2}(\Omega) \cap \dot{H}^{1}(\Omega)$ of the associated Dirichlet problem, defined by

$$
a(T f, v)=(f, v) \text { for all } v \in \dot{H}^{1}(\Omega) .
$$

$T$ has a discrete spectrum of eigenvalues $\left\{\mu_{j}\right\}_{j \geqslant 1}$, where $\mu_{j}=\lambda_{j}^{-1}$.

Following [2], we set $L^{2}=L^{2}(\Omega) \times L^{2}(\Omega)$ and define

$$
\begin{gathered}
T=\left(\begin{array}{cc}
0 & T \\
-I & 0
\end{array}\right): \mathrm{L}^{2} \rightarrow \mathrm{L}^{2}, \\
L=\left(\begin{array}{cc}
0 & -I \\
L & 0
\end{array}\right), \\
U(t)=\left(\begin{array}{c}
u(t) \\
D_{t} u(t)
\end{array}\right), \quad t \geqslant 0, \quad \text { and } U^{0}=\left(\begin{array}{c}
u^{0} \\
u_{t}^{0}
\end{array}\right) .
\end{gathered}
$$

Then (1.1) is equivalent to

$$
\left\{\begin{array}{l}
T D_{t} U+U=0, \quad 0<t \leqslant t^{*} \\
U(0)=U^{0}
\end{array}\right.
$$

or

$$
\left\{\begin{array}{l}
D_{t} U+L U=0, \quad 0<t \leqslant t^{*} \\
U(0)=U^{0}
\end{array}\right.
$$

For the spatial discretization we assume the existence of a one-parameter family $\left\{S_{h}(\Omega)\right\}_{0<h<1}$ of finite-dimensional subspaces of $L^{2}(\Omega)$, and corresponding operators $T_{h}: L^{2}(\Omega) \rightarrow S_{h}(\Omega)$ which approximate $T$ in the following sense:

(1.7) $T_{h}$ is selfadjoint, positive semidefinite on $L^{2}(\Omega)$ and positive definite on $S_{h}(\Omega)$.

(1.8) There exists an integer $r \geqslant 2$ and a constant $C$ such that $\left\|\left(T_{h}-T\right) f\right\| \leqslant$ $C h^{S+2}\|f\|_{S}$, for all $f \in \dot{H}^{S}(\Omega),-1 \leqslant S \leqslant r-2$. 
(1.9) There exists an $h_{0}>0$ and a function $\gamma(h)$ such that for $0<h \leqslant h_{0}$, $\left|\left(T_{h}-T\right) f\right| \leqslant \gamma(h)|T f|_{r}$, for all sufficiently smooth $f$.

(1.10) There exists a constant $C$ such that for all sufficiently smooth $f,\left|T_{h} f\right| \leqslant$ $C|T f|_{1}$, and $\left\|T_{h} f\right\| \leqslant C\|T f\|_{1}$.

(1.11) $T_{h}$ has a discrete spectrum of eigenvalues $\left\{0, \mu_{1}^{h}, \ldots, \mu_{M}^{h}\right\}$, in nondecreasing order, for some integer $M=M(h)$, with $\mu_{1}^{h}>0$. Moreover, for some $h_{0}>0$, if $h \leqslant h_{0}$, then $\mu_{M}^{h} \leqslant A$ for some constant $A$ independent of $h$.

From [2]-[4], it is known that, for example, for the standard Galerkin method using finite element subspaces $S_{h}(\Omega) \subset \dot{H}^{1}(\Omega)$, satisfying

$$
\inf _{\chi \in S_{h}(\Omega)}\left(\|w-\chi\|+h\|w-\chi\|_{1}\right) \leqslant C h^{s}\|w\|_{s} \quad \text { for } 1 \leqslant s \leqslant r
$$

with $T_{h}$ given by

$$
a\left(T_{h} f, \chi\right)=(f, \chi) \text { for all } \chi \in S_{h}(\Omega),
$$

(1.7), (1.8), (1.11) hold.

In [11] Nitsche shows for the standard Galerkin method in the constant coefficient case $L=-\Delta$, for $r>2$, under certain regularity assumptions on the triangulation defining $S_{h}$, that (1.9) and (1.10) hold for $\gamma(h)=C h^{r}$, for some constant $C$. In the case of Neumann boundary conditions (not considered here), Scott [14], shows that for $N=2,(1.9)$ and (1.10) hold with

$$
\gamma(h)= \begin{cases}C h^{r}, & r>2, \\ C h^{2} \ln h^{-1}, & r=2,\end{cases}
$$

for some constant $C$. For analogous results in the variable coefficient case (and the nonlinear case, not considered here), cf. the works of Rannacher [13], Nitsche [12], Frehse and Rannacher [9], among others.

As in [2], the semidiscrete approximation is a mapping $u_{h}:\left[0, t^{*}\right] \rightarrow S_{h}(\Omega)$ defined as follows. We set $S_{h}=S_{h}(\Omega) \times S_{h}(\Omega)$,

and

$$
V(t)=\left(\begin{array}{c}
u_{h}(t) \\
D_{t} u_{h}(t)
\end{array}\right), \quad t \geqslant 0,
$$

$$
T_{h}=\left(\begin{array}{cc}
0 & T_{h} \\
-I & 0
\end{array}\right): \quad \mathbf{L}^{2} \rightarrow \mathbf{L}^{2}
$$

Then $V$ is required to satisfy

$$
\begin{cases}T_{h} D_{t} V+V=0, & 0<t \leqslant t^{*} \\ V(0) & \text { given in } S_{h}\end{cases}
$$

In Theorem 2.1 we prove that if the initial values $V(0)$ in $(1.15)$ are chosen by 


$$
V(0)=T_{h}^{2 S} L^{2 S_{U^{0}}}=\left(\begin{array}{c}
T_{h}^{S} L^{S} u^{0} \\
T_{h}^{S} L^{S} u_{t}^{0}
\end{array}\right),
$$

with $S \geqslant[N / 2]+1$, then

$$
\sup _{0 \leqslant t \leqslant t^{*}}\left|u(t)-u_{h}(t)\right| \leqslant C\left\{\gamma(h)\left\|U^{0}\right\|_{S_{0}}+h^{r}\left\|U^{0}\right\|_{2 S+r+1}\right\},
$$

where $S_{0}=2 S+r+[N / 2]-1$.

In the standard Galerkin method (1.12), it is easily seen that the computation of $V(0)$ in (1.16) involves the solution of $2 \mathrm{~S}$ linear systems of equations with the same real matrix, and in such a case, with sufficiently smooth initial data $u^{\mathbf{0}}, u_{t}^{\mathbf{0}}$, we attain the optimal $L^{\infty}(\Omega)$-convergence.

In the same theorem we prove that if the operators $T_{h}$ are such that

$$
\mu_{1}^{h} \geqslant C h^{2},
$$

for some constant $C$ independent of $h$, and $V(0)$ is chosen by (1.16), then

$$
\sup _{0 \leqslant t \leqslant t^{*}}\left|D_{t} u(t)-D_{t} u_{h}(t)\right| \leqslant C\left\{\gamma(h)\left\|U^{0}\right\|_{S_{0}+1}+h^{r-1}\left\|U^{0}\right\|_{2 S+r+1}\right\}
$$

with $S$ and $S_{0}$ as above. It is known in the case where $T_{h}$ is defined by (1.12), (1.18) follows with an "inverse property" of the form

$$
\|\chi\|_{1} \leqslant C h^{-1}\|\chi\| \text { for all } \chi \in S_{h}(\Omega),
$$

for some constant $C$ independent of $h$.

In Theorem 2.2 we prove that if, alternatively, $V(0)$ in (1.15) is chosen by

$$
V(0)=T_{h}^{2 S+1} L^{2 S+1} U^{0}=\left(\begin{array}{c}
T_{h}^{S+1} L^{S+1} u^{0} \\
T_{h}^{S} L^{S} u_{t}^{0}
\end{array}\right),
$$

with $S \geqslant[N / 2]+1$ (same as above), then the optimal estimate (1.17) holds. Moreover, (1.19) is improved to

$$
\sup _{0 \leqslant t \leqslant t^{*}}\left|D_{t} u(t)-D_{t} u_{h}(t)\right| \leqslant C\left\{\gamma(h)\left\||| U^{0}\right\|_{S_{0}+1}+h^{r} \mid\left\|U^{0}\right\| \|_{2 S+r+2}\right\},
$$

without the assumption (1.18). Thus, by solving one extra linear system as dictated by (1.20), in comparison with (1.16), we obtain also optimal $L^{\infty}(\Omega)$-convergence for the time derivatives.

We now turn to single-step fully discrete approximations to (1.1), following [2]. Let $r$ be a rational function of the complex variable $w=x+i y$ which satisfies for constants $\sigma>0, C<\infty$ and $\nu>0$

$$
\left|r(i y)-e^{-i y}\right| \leqslant C|y|^{\nu+1}, \quad|y| \leqslant \sigma .
$$

Definition I. $r$ is defined to be of class $\mathrm{i}-1$ if in addition to (1.22),

$$
|r(i y)| \leqslant 1 \quad \text { for }|y| \leqslant \alpha,
$$

for some constant $\alpha>0$. 
Definition II. $r$ is defined to be of class i-II if $r$ satisfies (1.22) and (1.23) with $\alpha=\infty$.

For convenience we set $L_{h}=T_{h}^{-1}: S_{h}(\Omega) \rightarrow S_{h}(\Omega)$, and consequently

$$
L_{h}=\left(\begin{array}{cc}
0 & -I \\
L_{h} & 0
\end{array}\right)
$$

Then (1.15) is equivalent to

$$
\begin{cases}D_{t} V+L_{h} V=0, & 0<t \leqslant t^{*} \\ V(0) & \text { given in } S_{h} .\end{cases}
$$

For a chosen discrete time step $k>0$, we have from (1.25),

$$
V(t+k)=\exp \left(-k L_{h}\right) V(t), \quad t \geqslant 0,
$$

which in turn motivates the following fully discrete approximations. For

is sought satisfying

$$
n=0,1, \ldots,\left[t^{*} / k\right], \quad W^{n}=\left(\begin{array}{c}
W_{1}^{n} \\
W_{2}^{n}
\end{array}\right) \in S_{h}
$$

$$
\left\{\begin{array}{l}
W^{n+1}=r\left(k L_{h}\right) W^{n}, \quad n \geqslant 0, \\
W^{0}=V(0) .
\end{array}\right.
$$

A computationally efficient class of rational functions $r_{\nu}$ of class i-II for any even integer choice of $\nu$ is constructed in [2]. It is shown that the approximation $W^{n+1}$ is obtained from $W^{n}$ in (1.26) with the solution of $v$ linear systems of equations, with the same real matrix. Hence, a single $L U$ decomposition of this matrix is required; and a fixed number, $\nu$, of "back solves" need be carried out at each time step. Other examples such as Padé approximations are also discussed in [2], [7].

In Theorem 3.1 we derive the following $L^{\infty}(\Omega)$-estimate. It will be assumed that there exists an integer $J_{0}$ and an $h_{0}>0$ such that

$$
\sum_{j}\left(\mu_{j}^{h}\right)^{J_{0}} \leqslant C<\infty
$$

for some constant $C$. independent of $h$, for all $h \leqslant h_{0}$.

In the case of the standard Galerkin method (1.12) it can be readily deduced that (1.27) holds for some finite $J_{0}=J_{0}(\Omega)$, by virtue of the fact that $\left\{\left(\mu_{j}^{h}\right)^{-1}\right\}$ approximates the spectrum of the elliptic operator $L$, for $h$ sufficiently small. See Section 4 for details.

If $r$ is of class $\mathrm{i}-\mathrm{II}$, and $w^{0}=V(0)$ is chosen by (1.16), with $S \geqslant[N / 2]+J_{0}+1$, we prove that

$$
\begin{aligned}
\max _{0 \leqslant n \leqslant\left[t^{*} / k\right]}\left|W_{1}^{n}-u(n k)\right| \\
\leqslant C\left\{\gamma(h)\left\|U^{0}\right\|_{S_{0}}+h^{r}\left\|U^{0}\right\|_{2 S+r+1}+k^{\nu}\left\|U^{0}\right\|_{2 S+\nu+1}\right\},
\end{aligned}
$$

where $S_{0}=2 S+r+[N / 2]-1$. 
In addition to the above conditions, if $(1.18)$ holds and $k / h \leqslant C$ for any constant $C>0$, we prove that

$$
\begin{aligned}
& \max _{0 \leqslant n \leqslant\left[t^{*} / k\right]}\left|W_{2}^{n}-D_{t} u(n k)\right| \\
& \quad \leqslant C\left\{\gamma(h)\left\|U^{0}\right\|_{S_{0}+1}+h^{r-1}\left\|U^{0}\right\|_{2 S+r+1}+k^{\nu-1}\left\|U^{0}\right\| \|_{2 S+\nu+1}\right\},
\end{aligned}
$$

with $S$ and $S_{0}$ as above.

In Theorem 3.2 we prove that for $r$ of class i-II, and $W^{0}=V(0)$ chosen by $(1.20)$,

$$
\begin{aligned}
& \max _{0 \leqslant n \leqslant\left[t^{*} / k\right]}\left|W_{1}^{n}-u(n k)\right| \\
& \quad \leqslant C\left\{\gamma(h)\left\|U^{0}\right\|_{S_{0}}+h^{r}\left\|U^{0}\right\|_{2 S+r+2}+k^{\nu}\left\|U^{0}\right\|_{2 S+\nu+2}\right\}
\end{aligned}
$$

and

$$
\begin{aligned}
& \max _{0 \leqslant n \leqslant\left[t^{*} / k\right]}\left|W_{2}^{n}-D_{t} u(n k)\right| \\
& \quad \leqslant C\left\{\gamma(h)\left\|U^{0}\right\|_{S_{0}+1}+h^{r}\left\|\mid U^{0}\right\|_{2 S+r+2}+k^{\nu}\left\|U^{0}\right\|_{2 S+\nu+2}\right\},
\end{aligned}
$$

without the extra assumption (1.18).

Again it is seen that in order to obtain the optimal $L^{\infty}(\Omega)$-convergence it is sufficient to regulate the starting values by performing a given number of projections of the given initial data $u^{0}$ and $u_{t}^{0}$. In particular, to obtain (1.30) and (1.31) it suffices to solve the $2 S+1$ linear systems with the same real matrix as dictated by (1.20), with $S=[N / 2]+J_{0}+1$.

For $r$ of class $\mathrm{i}-\mathrm{I}$, we show that there exists a constant $C=C(\alpha)$, such that with the Courant-type condition $k / h \leqslant C$, and assumption (1.18), the estimates (1.28) and (1.29) hold, for $W^{0}=V(0)$ chosen by (1.16), and for $W^{0}=V(0)$ chosen by (1.20), the estimates (1.30) and (1.31) hold.

Section 2 is devoted to the derivation of the $L^{\infty}(\Omega)$-error estimates for the semidiscrete approximation, (1.17), (1.19) and (1.21). In Section 3 we derive the estimates (1.28)-(1.31) for the fully discrete approximation. Throughout the remainder of the paper, no distinction will be made between the different constants appearing in the error bounds, and all will be denoted by the generic $C$. Also, conditions of the type $h \leqslant h_{0}$ will be implicitly assumed wherever needed.

2. $L^{\infty}$-Estimates for Semidiscrete Approximations. We first modify slightly the notation of [2]. On $\mathbf{L}^{2}$ we define the positive semidefinite bilinear form, $((\cdot, \cdot))_{h}$, as follows. For

$$
\Phi=\left(\begin{array}{l}
\varphi_{1} \\
\varphi_{2}
\end{array}\right), \quad \Psi=\left(\begin{array}{l}
\psi_{1} \\
\psi_{2}
\end{array}\right) \in \mathbf{L}^{2},
$$

$((\Phi, \Psi))_{h}=\left(\varphi_{1}, \psi_{1}\right)+\left(T_{h} \varphi_{2}, \psi_{2}\right) . \quad((\cdot, \cdot))_{h}$ is an inner product on $S_{h}$. The associated norm we denote by $\|\mid \cdot\| \|_{h}$, i.e., $\|\mid \Phi\|_{h}=((\Phi, \Phi))_{h}^{1 / 2}$, which is a seminorm on $\mathbf{L}^{2}$. 
We point out that no confusion should arise between the norm $\|\cdot\| \|_{h}$ and the norms $\|\cdot\|_{S}, S \geqslant 1$, defined in Section 1 .

$P$ will denote the $L^{2}(\Omega)$-projection operator onto $S_{h}(\Omega)$ and $P$ the induced $\mathbf{L}^{2}$-projection operator onto $S_{h}$.

Let $\psi_{j}^{h} \in S_{h}(\Omega)$ denote the eigenfunction of $T_{h}$ corresponding to the positive eigenvalue $\mu_{j}^{h}$, i.e., $T_{h} \psi_{j}^{h}=\mu_{j}^{h} \psi_{j}^{h}, j=1,2, \ldots, M$, chosen so that the set $\left\{\psi_{j}^{h}\right\}_{j=1}^{M}$ is orthonormal in $L^{2}(\Omega)$.

We note that $L_{h}$ is defined on $L^{2}(\Omega)$ by the spectral representation

$$
L_{h} v=\sum_{j}\left(\mu_{j}^{h}\right)^{-1}\left(v, \psi_{j}^{h}\right) \psi_{j}^{h}, \quad v \in L^{2}(\Omega)
$$

By Parseval's relation we then see that

$$
\left\|L_{h}\right\| \leqslant \sup _{j}\left(\mu_{j}^{h}\right)^{-1}
$$

Hence, if (1.18) holds, we have that

$$
\left\|L_{h}\right\| \leqslant C h^{-2} \text {. }
$$

As a consequence of the definition of $L_{h}$, if $T_{h}$ and $L_{h}$ are defined by (1.14) and (1.24), respectively, a simple computation gives, for integer $S$,

$$
L_{h}^{S} T_{h}^{S}=T_{h}^{S} L_{h}^{S}=P \quad \text { if } S>1
$$

and

$$
L_{h} T_{h}=\left(\begin{array}{ll}
I & 0 \\
0 & P
\end{array}\right), \quad T_{h} L_{h}=\left(\begin{array}{cc}
P & 0 \\
0 & I
\end{array}\right)
$$

Of course, it is easily seen that for all positive integers $S, L^{S} T^{S}$ is the identity on $\mathrm{L}^{2}$ and that $T^{S} L^{S}$ is the identity on $\dot{H}^{S+1} \times \dot{H}^{S-1}$, if $S$ is odd, and on $\dot{H}^{S} \times \dot{H}^{S}$ if $S$ is even.

We set $E=U-V$, where $U$ is the solution of (1.5) and $V$ is the solution of (1.15) . To obtain the maximum norm estimates for $u-u_{h}$ we first derive estimates of the even-order time derivatives of $E$ in the seminorm $\|\cdot\|_{h}$.

LEMMA 2.1. For $S \geqslant 0$ integer, let the initial condition in (1.15) be given by $V(0)=T_{h}^{2 S} L^{2 S} U^{0}$ if $S>0$ and $V(0)=P U^{0}$ if $S=0$. Then for some constant $C=C\left(t^{*}\right)$,

$$
\sup _{0 \leqslant t \leqslant t^{*}}\left\|D_{t}^{2 S} E(t)\right\|_{h} \leqslant C h^{r}\left[\left\|u^{0}\right\|_{2 S+r+1}+\left\|u_{t}^{0}\right\|_{2 S+r}\right]
$$

Proof. By (1.5) and (1.15) we see that $E$ satisfies

$$
T_{h} D_{t} E+E=\left(T_{h}-T\right) D_{t} U \text { for } 0<t \leqslant t^{*},
$$

which implies that

$$
T_{h} D_{t}^{2 S+1} E+D_{t}^{2 S} E=\left(T_{h}-T\right) D_{t}^{2 S+1} U
$$


Hence

$$
\begin{aligned}
\left(\left(T_{h} D_{t}^{2 S+1}\right.\right. & \left.\left.E(t), D_{t}^{2 S+1} E(t)\right)\right)_{h}+\frac{1}{2} \frac{d}{d t}\left\|D_{t}^{2 S} E(t)\right\|_{h}^{2} \\
= & \left(\left(\left(T_{h}-T\right) D_{t}^{2 S+1} U(t), D_{t}^{2 S+1} E(t)\right)\right)_{h} \\
= & \frac{d}{d t}\left(\left(\left(T_{h}-T\right) D_{t}^{2 S+1} U(t), D_{t}^{2 S} E(t)\right)\right)_{h} \\
& -\left(\left(\left(T_{h}-T\right) D_{t}^{2 S+2} U(t), D_{t}^{2 S} E(t)\right)\right)_{h}
\end{aligned}
$$

Integrating the last equation and using the fact that $\left(\left(T_{h} \Phi, \Phi\right)\right)_{h}=0$ for any $\Phi \in \mathrm{L}^{2}$, we see that

$$
\begin{aligned}
\left\|D_{t}^{2 S} E(t)\right\|_{h}^{2}= & \left\|D_{t}^{2 S} E(0)\right\|_{h}^{2}+2\left(\left(\left(T_{h}-T\right) D_{t}^{2 S+1} U(t), D_{t}^{2 S} E(t)\right)\right)_{h} \\
& -2\left(\left(\left(T_{h}-T\right) D_{t}^{2 S+1} U(0), D_{t}^{2 S} E(0)\right)\right)_{h} \\
& -2 \int_{0}^{t}\left(\left(\left(T_{h}-T\right) D_{\tau}^{2 S+2} U(\tau), D_{\tau}^{2 S} E(\tau)\right)\right)_{h} d \tau
\end{aligned}
$$

Now, using (1.6) and (1.25), we see that

$$
D_{t}^{2 S} E(0)=L^{2 S} U^{0}-L_{h}^{2 S} V(0) .
$$

Hence, since $V(0)=T_{h}^{2 S} L^{2 S} U^{0}, S>0$, we see that

$$
D_{t}^{2 S} E(0)=L^{2 S} U^{0}-L_{h}^{2 S} T_{h}^{2 S} L^{2 S} U^{0}=(I-P) L^{2 S} U^{0}, \quad S \geqslant 0 .
$$

Since

the above equation becomes

$$
L^{2 S}=(-1)^{S}\left(\begin{array}{cc}
L^{S} & 0 \\
0 & L^{S}
\end{array}\right)
$$

and thus

$$
D_{t}^{2 S} E(0)=(-1)^{s}\left(\begin{array}{c}
(I-P) L^{S} u^{0} \\
(I-P) L^{S} u_{t}^{0}
\end{array}\right)
$$

$$
\left\|D_{t}^{2 S} E(0)\right\|_{h}=\left\|(I-P) L^{S} u^{0}\right\| .
$$

Now, using (2.5), we have,

$$
\begin{aligned}
\left(\left(\left(T_{h}-T\right) D_{t}^{2 S+1} U^{0}, D_{t}^{2 S} E(0)\right)\right)_{h} \\
\quad=(-1)^{S}\left(\left(T_{h}-T\right) D_{t}^{2 S+2} u(0),(I-P) L^{S} u^{0}\right) \\
\quad=(-1)^{S+1}\left(T D_{t}^{2 S+2} u(0),(I-P) L^{S} u^{0}\right) \\
\quad=(-1)^{S+2}\left(D_{t}^{2 S} u(0),(I-P) L^{S} u^{0}\right) \\
\quad=(-1)^{S+2}\left(D_{t}^{2 S}\left(u(0)-u_{h}(0)\right),(I-P) L^{S} u^{0}\right) \\
\quad=(-1)^{S+2}\left(\left(D_{t}^{2 S} E(0)\right)_{1},(I-P) L^{S} u^{0}\right) \\
\quad=\left\|(I-P) L^{S} u^{0}\right\|^{2}
\end{aligned}
$$


From (2.3), (2.6), (2.7) we obtain

$$
\begin{aligned}
\left\|D_{t}^{2 S} E(t)\right\|_{h}^{2} \leqslant & 2\left(\left(\left(T_{h}-T\right) D_{t}^{2 S+1} U(t), D_{t}^{2 S} E(t)\right)\right)_{h} \\
& \quad-2 \int_{0}^{t}\left(\left(\left(T_{h}-T\right) D_{\tau}^{2 S+2} U(\tau), D_{\tau}^{2 S} E(\tau)\right)\right)_{h} d \tau \\
\leqslant & \frac{1}{4}\left\|D_{t}^{2 S} E(t)\right\|_{h}^{2}+4\left\|\left(T_{h}-T\right) D_{t}^{2 S+1} U(t)\right\|_{h}^{2} \\
& +\frac{1}{4} \sup _{0 \leqslant t \leqslant t^{*}}\left\|D_{t}^{2 S} E(t)\right\|_{h}^{2}+4 t^{*} \int_{0}^{t^{*}}\left\|\left(T_{h}-T\right) D_{\tau}^{2 S+2} U(\tau)\right\|_{h}^{2} d \tau .
\end{aligned}
$$

Now, by (1.8)

$$
\begin{aligned}
\left\|\left(T_{h}-T\right) D_{t}^{2 S+1} U(t)\right\|_{h} & =\left\|\left(T_{h}-T\right) D_{t}^{2 S+2} u(t)\right\| \leqslant C h^{r}\left\|D_{t}^{2 S+2} u(t)\right\|_{r-2} \\
& =C h^{r}\left\|L^{S+1} u(t)\right\|_{r-2} \leqslant C h^{r}\|u(t)\|_{2 S+r}
\end{aligned}
$$

Similarly,

$$
\left\|\left(T_{h}-T\right) D_{t}^{2 S+2} U(t)\right\|_{h} \leqslant C h^{r}\left\|D_{t} u(t)\right\|_{2 S+r}
$$

Then, by the above, (1.2) and (2.8) give (2.2).

We also need the following result in the case that the initial conditions are chosen by (1.20).

LEMMA 2.2. For $S \geqslant 0$ integer, let the initial condition in (1.15) be given by $V(0)=T_{h}^{2 S+1} L^{2 S+1} U^{0}$ if $S>0$ and $V(0)=P T_{h} L U^{0}$ if $S=0$. Then, for some constant $C=C\left(t^{*}\right)$ :

$$
\sup _{0 \leqslant t \leqslant t^{*}}\left\|D_{t}^{2 S} E(t)\right\|_{h} \leqslant C h^{r}\left(\left\|u^{0}\right\|_{2 S+r+1}+\left\|u_{t}^{0}\right\|_{2 S+r}\right)
$$

Proof. We need merely modify the proof of Lemma 2.1 to account for the different starting values. Obviously (2.3) and (2.4) still hold. By the hypothesis on $V(0),(2.4)$ gives

$$
D_{t}^{2 S} E(0)=\left(T-P T_{h}\right) L^{2 S+1} U^{0}, \quad S \geqslant 0 .
$$

Since

we conclude that

$$
L^{2 S+1}=(-1)^{S}\left(\begin{array}{cc}
0 & -L^{S} \\
L^{S+1} & 0
\end{array}\right)
$$

$$
D_{t}^{2 S} E(0)=(-1)^{S}\left(\begin{array}{c}
\left(T-T_{h}\right) L^{S+1} u^{0} \\
(I-P) L^{S} u_{t}^{0}
\end{array}\right)
$$

Hence

$$
\left\|D_{t}^{2 S} E(0)\right\|_{h}=\left\|\left(T-T_{h}\right) L^{S+1} u^{0}\right\|
$$


Now, using (2.10), we obtain

$$
\begin{aligned}
\left(\left(\left(T_{h}-T\right) D_{t}^{2 S+1} U(0), D_{t}^{2 S} E(0)\right)\right)_{h} \\
\quad=(-1)^{S}\left(\left(T_{h}-T\right) D_{t}^{2 S+2} u(0),\left(T-T_{h}\right) L^{S+1} u^{0}\right) \\
\quad=(-1)^{2 S+1}\left(\left(T_{h}-T\right) L^{S+1} u(0),\left(T-T_{h}\right) L^{S+1} u^{0}\right) \\
\quad=\left\|\left(T-T_{h}\right) L^{S+1} u^{0}\right\|^{2} .
\end{aligned}
$$

Using (2.11) and (2.12) in (2.3), we obtain

$$
\begin{aligned}
\left\|D_{t}^{2 S} E(t)\right\|_{h}^{2}= & 2\left(\left(\left[T_{h}-T\right] D_{t}^{2 S+1} U(t), D_{t}^{2 S} E(t)\right)\right)_{h} \\
& -2 \int_{0}^{t}\left(\left(\left[T_{h}-T\right] D_{\tau}^{2 S+2} U(\tau), D_{\tau}^{2 S} E(\tau)\right)\right)_{h} d \tau-\left\|\left(T-T_{h}\right) L^{S+1} u^{0}\right\|^{2}
\end{aligned}
$$

The result (2.9) now follows from this last equation via the same arguments used in Lemma 2.1.

We now set $Z=D_{t} U-D_{t} V=D_{t} E$. To obtain maximum norm estimates for $D_{t} u-D_{t} u_{h}$ we first derive estimates of the even-order time derivatives of $Z$ in the seminorm \|\|$\cdot\|\|_{h}$.

LEMMA 2.3. For $S \geqslant 0$ integer, let $V(0)=T_{h}^{2 S} L^{2 S} U^{0}$ if $S>0$ and $V(0)=$ $P U^{0}$ if $S=0$. In addition let $T_{h}$ satisfy (1.18). Then, for some constant $C=C\left(t^{*}\right)$ :

$$
\sup _{0 \leqslant t \leqslant t^{*}}\left\|D_{t}^{2 S} Z(t)\right\|_{h} \leqslant C h^{r-1}\left(\left\|u^{0}\right\|_{2 S+r+1}+\left\|u_{t}^{0}\right\|_{2 S+r}\right) .
$$

Proof. Clearly $Z$ satisfies

$$
T_{h} D_{t} Z+Z=\left(T_{h}-T\right) D_{t}^{2} U, \quad 0<t \leqslant t^{*},
$$

which implies that

$$
T_{h} D_{t}^{2 S+1} Z+D_{t}^{2 S} Z=\left(T_{h}-T\right) D_{t}^{2 S+2} U, \quad 0<t \leqslant t^{*}
$$

Hence

$$
\begin{aligned}
\left\|D_{t}^{2 S} Z(t)\right\|_{h}^{2}= & \left\|D_{t}^{2 S} Z(0)\right\|_{h}^{2}+2\left(\left(\left(T_{h}-T\right) D_{t}^{2 S+2} U(t), D_{t}^{2 S} Z(t)\right)\right)_{h} \\
& -2\left(\left(\left(T_{h}-T\right) D_{t}^{2 S+2} U(0), D_{t}^{2 S} Z(0)\right)\right)_{h} \\
& -2 \int_{0}^{t}\left(\left(\left(T_{h}-T\right) D_{\tau}^{2 S+3} U(\tau), D_{\tau}^{2 S} Z(\tau)\right)\right)_{h} d \tau
\end{aligned}
$$

Now, it is easily seen that

$$
D_{t}^{2 S} Z(0)=-L^{2 S+1} U^{0}+L_{h}^{2 S+1} V(0) .
$$

By the given initial conditions then,

$$
D_{t}^{2 S} Z(0)=\left(L_{h} P-L\right) L^{2 S} U^{0}, \quad S \geqslant 0 .
$$

Since by the definition of $L_{h}, L_{h} P=P L_{h}=L_{h}$, we have

$$
D_{t}^{2 S_{Z}} Z(0)=(-1)^{S}\left(\begin{array}{c}
(I-P) L^{S_{u}^{0}} \\
\left(L_{h}-L\right) L^{S_{u}^{0}}
\end{array}\right)
$$


Thus

$$
\begin{aligned}
\left\|D_{t}^{2 S} Z(0)\right\|_{h}^{2} & =\left\|(I-P) L^{S} u_{t}^{0}\right\|^{2}+\left(T_{h}\left[L_{h}-L\right] L^{S} u^{0},\left[L_{h}-L\right] L^{S} u^{0}\right) \\
& =\left\|(I-P) L^{S} u_{t}^{0}\right\|^{2}+\left(\left[P-T_{h} L\right] L^{S} u^{0}, L_{h}\left[P-T_{h} L\right] L^{S} u^{0}\right)
\end{aligned}
$$

Also, using (2.16), we see that

$$
\begin{aligned}
\left(\left(\left(T_{h}-T\right) D_{t}^{2 S+2} U(0), D_{t}^{2 S} Z(0)\right)\right)_{h} \\
\quad=(-1)^{S}\left(\left(T_{h}-T\right) D_{t}^{2 S+3} u(0),(I-P) L^{S} u_{t}^{0}\right) \\
\quad=(-1)^{S+1}\left(T D_{t}^{2 S+3} u(0),(I-P) L^{S} u_{t}^{0}\right) \\
\quad=(-1)^{S+2}\left(D_{t}^{2 S+1} u(0),(I-P) L^{S} u_{t}^{0}\right) \\
\quad=(-1)^{S+2}\left(D_{t}^{2 S}\left(D_{t} u(0)-D_{t} u_{h}(0)\right),(I-P) L^{S} u_{t}^{0}\right) \\
\quad=(-1)^{S+2}\left(\left(D_{t}^{2 S} Z(0)\right)_{1},(I-P) L^{S} u_{t}^{0}\right) \\
\quad=\left\|(I-P) L^{S} u_{t}^{0}\right\|^{2} .
\end{aligned}
$$

Now, using (2.1) in (2.17), we obtain

$$
\begin{aligned}
((P- & \left.\left.T_{h} L\right) L^{S} u^{0}, L_{h}\left(P-T_{h} L\right) L^{S} u^{0}\right) \leqslant\left\|L_{h}\right\|\left\|\left(P-T_{h} L\right) L^{S} u^{0}\right\|^{2} \\
& \leqslant\left\|L_{h}\right\|\left\|\left(I-T_{h} L\right) L^{S} u^{0}\right\|^{2} \leqslant C h^{-2}\left\|\left(T-T_{h}\right) L^{S+1} u^{0}\right\|^{2} \\
& \leqslant C h^{2 r-2}\left\|u^{0}\right\|_{2 S+r}^{2} .
\end{aligned}
$$

Hence, (2.14), (2.17), (2.18) and the last inequality imply that

$$
\begin{aligned}
\left\|D_{t}^{2 S} Z(t)\right\|_{h}^{2} \leqslant & C h^{2 r-2}\left\|u^{0}\right\|_{2 S+r}^{2}+2\left(\left(\left(T_{h}-T\right) D_{t}^{2 S+2} U(t), D_{t}^{2 S} Z(t)\right)\right)_{h} \\
& -2 \int_{0}^{t}\left(\left(\left(T_{h}-T\right) D_{\tau}^{2 S+3} U(\tau), D_{\tau}^{2 S} Z(\tau)\right)\right)_{h} d \tau,
\end{aligned}
$$

from which (2.13) follows easily by estimating the inner products in the right-hand side of the above equation in an analogous way as in Lemma 2.1 .

The analogous result in the case of the initial condition (1.20) is given by:

LEMMA 2.4. For $S \geqslant 0$ integer, let $V(0)=T_{h}^{2 S+1} L^{2 S+1} U^{0}$ if $S>0$ and $V(0)$ $=P T_{h} L U^{0}$ if $S=0$. Then, for some constant $C=C\left(t^{*}\right)$ :

$$
\sup _{0 \leqslant t \leqslant t^{*}}\left\|D_{t}^{2 S} Z(t)\right\|_{h} \leqslant C h^{r}\left(\left\|u^{0}\right\|_{2 S+r+2}+\left\|u_{t}^{0}\right\|_{2 S+r+1}\right) \text {. }
$$

Proof. Obviously, (2.14) and (2.15) still hold. Putting the appropriate $V(0)$ in (2.15), we obtain

$$
D_{t}^{2 S} Z(0)=(-1)^{S+1}\left(\begin{array}{c}
-(I-P) L^{S} u_{t}^{0} \\
(I-P) L^{S+1} u^{0}
\end{array}\right)
$$

which gives

$$
\left\|D_{t}^{2 S} Z(0)\right\|_{h}=\left\|(I-P) L^{S} u_{t}^{0}\right\|
$$


As in Lemma 2.3, we see that in this case, also,

$$
\left(\left(\left(T_{h}-T\right) D_{t}^{2 S+2} U(0), D_{t}^{2 S} Z(0)\right)\right)_{h}=\left\|(I-P) L^{S} u_{t}^{0}\right\|^{2}
$$

In analogy with previous calculations, (2.14), (2.21) and (2.22) give then the estimate (2.19).

To derive now the maximum norm estimates we recall a result from [4]. We denote by $\|\cdot\|_{L^{p}}$ the norm

$$
\|v\|_{L^{p}}=\left(\int_{\Omega}|v|^{p} d x\right)^{1 / p}
$$

on $L^{p}(\Omega)$, for $1 \leqslant p<\infty$.

Lemma 2.5. Let $2 \leqslant p \leqslant \infty$ and $0<1 / q-1 / p<1 / N$. Then there exists $a$ constant $C$ such that for every $w \in L^{q}$

$$
\left\|T_{h} w\right\|_{L} \leqslant C\|w\|_{L} q
$$

A proof of Lemma 2.5 is given in [4].

The maximum norm estimate will depend on the following result:

Lemma 2.6. Let $\gamma(h)$ be defined by (1.9), and let $S$ be a given integer such that $S \geqslant[N / 2]+1$. If $S_{0}=2 S+r+[N / 2]-1$, then, for some constant $C$,

$$
\begin{aligned}
& \sup _{0 \leqslant t \leqslant t^{*}}\left|u(t)-u_{h}(t)\right| \\
& \quad \leqslant C\left\{\gamma(h)\left(\left\|u^{0}\right\|_{S_{0}}+\left\|u_{t}^{0}\right\|_{S_{0^{-1}}}\right)+\sup _{0 \leqslant t \leqslant t^{*}}\left\|D_{t}^{2 S}\left(u(t)-u_{h}(t)\right)\right\|\right\}
\end{aligned}
$$

and

$$
\begin{aligned}
& \sup _{0 \leqslant t \leqslant t^{*}}\left|D_{t}\left(u(t)-u_{h}(t)\right)\right| \\
& \quad \leqslant C\left\{\gamma(h)\left(\left\|u^{0}\right\|_{S_{0}+1}+\left\|u_{t}^{0}\right\|_{S_{0}}\right)+\sup _{0 \leqslant t \leqslant t^{*}}\left\|D_{t}^{2 S+1}\left(u(t)-u_{h}(t)\right)\right\|\right\} .
\end{aligned}
$$

Proof. Let $e=u-u_{h}$. Then from (1.5) and (1.15), for $0<t \leqslant t^{*}$,

$$
T_{h} D_{t}^{2} e(t)+e(t)=\rho(t) \equiv\left(T_{h}-T\right) D_{t}^{2} u(t),
$$

from which it follows that, for integer $j$,

$$
D_{t}^{j} e(t)=D_{t}^{j} \rho(t)-T_{h} D_{t}^{j+2} e(t) .
$$

Hence, for $2 \leqslant p_{j} \leqslant \infty, 0<1 / p_{j+2}-1 / p_{j}<1 / N$,

$$
\begin{aligned}
\left\|D_{t}^{j} e(t)\right\|_{L} p_{j} & \leqslant\left\|D_{t}^{j} \rho(t)\right\|_{L} p_{j}+\left\|T_{h} D_{t}^{j+2} e(t)\right\|_{L} p_{j} \\
& =\left\|\left(T-T_{h}\right) D_{t}^{j+2} u(t)\right\|_{L} p_{j}+\left\|T_{h} D_{t}^{j+2} e(t)\right\|_{L} p_{j} .
\end{aligned}
$$

Now, using (1.9), we have that

$$
\begin{aligned}
\left\|\left(T-T_{h}\right) D_{t}^{j+2} u(t)\right\|_{L} p_{j} & \leqslant C\left|\left(T-T_{h}\right) D_{t}^{j+2} u(t)\right| \leqslant C \gamma(h)\left|T D_{t}^{j+2} u(t)\right|_{r} \\
& =C \gamma(h)\left|D_{t}^{j} u(t)\right|_{r} .
\end{aligned}
$$


Hence, (2.23) and (2.26) give

$$
\left\|D_{t}^{j} e(t)\right\|_{L} p_{j} \leqslant C\left(\gamma(h)\left|D_{t}^{j} u(t)\right|_{r}+\left\|D_{t}^{j+2} e(t)\right\|_{L} p_{j+2}\right) .
$$

It is easily seen that for $S \geqslant[N / 2]+1$ a decreasing sequence $\infty=p_{0}>p_{2}>$ $p_{4}>\cdots>p_{2 S}=2$ can be found such that $0<1 / p_{j+2}-1 / p_{j}<1 / N, 0 \leqslant j \leqslant$ $2 S-2$. Hence, using the inequality (2.27) recursively for $j=0,2, \ldots, 2 S-2$, we see that

$$
|e(t)| \leqslant C\left\{\gamma(h)\left(|u(t)|_{r}+\left|D_{t}^{2} u(t)\right|_{r}+\cdots+\left|D_{t}^{2 S-2} u(t)\right|_{r}\right)+\left\|D_{t}^{2 S} e(t)\right\|\right\} .
$$

Using (1.1), we can write the above as

$$
|e(t)| \leqslant C\left\{\gamma(h)|u(t)|_{r+2 S-2}+\left\|D_{t}^{2 S} e(t)\right\|\right\},
$$

which, by Sobolev's inequality and (1.2), gives (2.24). The proof of (2.25) is entirely analogous.

We now obtain the main results of this section.

THEOREM 2.1. Let $V(0)=T_{h}^{2 S} L^{2 S} U^{0}$, where $S$ is an integer such that $S \geqslant$ $[N / 2]+1$, and let $S_{0}=2 S+r+[N / 2]-1$. Then, for some constant $C=C\left(t^{*}\right)$, we have that

$$
\begin{aligned}
& \sup _{0 \leqslant t \leqslant t^{*}}\left|u(t)-u_{h}(t)\right| \\
& \quad \leqslant C\left\{\gamma(h)\left(\left\|u^{0}\right\|_{S_{0}}+\left\|u_{t}^{0}\right\|_{S_{0}-1}\right)+h^{r}\left(\left\|u^{0}\right\|_{2 s+r+1}+\left\|u_{t}^{0}\right\|_{2 S+r}\right)\right\}
\end{aligned}
$$

If in addition (1.18) holds, we also have

$$
\begin{aligned}
& \sup _{0 \leqslant t \leqslant t^{*}}\left|D_{t} u(t)-D_{t} u_{h}(t)\right| \\
& \quad \leqslant C\left\{\gamma(h)\left(\left\|u^{0}\right\|_{S_{0}+1}+\left\|u_{t}^{0}\right\|_{S_{0}}\right)+h^{r-1}\left(\left\|u^{0}\right\|_{2 S+r+1}+\left\|u_{t}^{0}\right\|_{2 S+r}\right)\right\} .
\end{aligned}
$$

Proof. It is immediately seen that (2.28) follows from (2.2), (2.24) and the fact that for $E=U-V$,

$$
\left\|D_{t}^{2 S}\left(u(t)-u_{h}(t)\right)\right\| \leqslant\left\|D_{t}^{2 S} E(t)\right\|_{h} .
$$

Similarly, (2.29) follows from (2.13) and (2.25).

As a consequence of Lemmas 2.2, 2.4, and 2.6 we also obtain

THEOREM 2.2. Let $V(0)=T_{h}^{2 S+1} L^{2 S+1} U^{0}$, where $S$ is an integer such that $S \geqslant[N / 2]+1$, and let $S_{0}=2 S+r+[N / 2]-1$. Then, for some constant $C=$ $C\left(t^{*}\right)$ we have that

$$
\begin{aligned}
& \sup _{0 \leqslant t<t^{*}}\left|u(t)-u_{h}(t)\right| \\
& \quad \leqslant C\left\{\gamma(h)\left(\left\|u^{0}\right\|_{S_{0}}+\left\|u_{t}^{0}\right\|_{s_{0}-1}\right)+h^{r}\left(\left\|u^{0}\right\|_{2 s+r+1}+\left\|u_{t}^{0}\right\|_{2 s+r}\right)\right\}
\end{aligned}
$$


and

$$
\begin{aligned}
& \sup _{0 \leqslant t \leqslant t^{*}}\left|D_{t} u(t)-D_{t} u_{h}(t)\right| \\
& \quad \leqslant C\left\{\gamma(h)\left(\left\|u^{0}\right\|_{S_{0}+1}+\left\|u_{t}^{0}\right\|_{S_{0}}\right)+h^{r}\left(\left\|u^{0}\right\|_{2 S+r+2}+\left\|u_{t}^{0}\right\|_{2 S+r+1}\right)\right\}
\end{aligned}
$$

Proof. The result (2.30) follows by using (2.9) in (2.24). (2.31) follows by using (2.19) in (2.24).

Remarks. (i) Theorem 2.1 basically asserts that in the case where the initial values of the semidiscrete approximation (1.15) are chosen via (1.16), one obtains optimal $L^{\infty}(\Omega)$-convergence for the error $u-u_{h}$. In addition, one obtains naturally a "consistent" suboptimal estimate, of $O\left(h^{r-1}\right)$, for the time derivative of the error $D_{t} u$ $-D_{t} u_{h}$, provided we work with space discretizations satisfying (1.18).

(ii) Theorem 2.2 shows that if one desires optimal $L^{\infty}(\Omega)$-convergence for both the error as well as for its time derivative, it is sufficient to choose the initial values of the semidiscrete approximation via (1.20). This amounts in practice to solving $2 S+1$ linear systems with the same real matrix, initially.

(iii) From Lemma 2.3 of this work we obtain a result which supplements the results of [1], [2] on $L^{2}(\Omega)$-convergence. With $S=0$ in Lemma 2.3 we have $V(0)=$ $P U^{0}$ (starting with $L^{2}(\Omega)$-projections), and under condition (1.18) we obtain

$$
\sup _{0 \leqslant t \leqslant t^{*}}\left\|D_{t} u(t)-D_{t} u_{h}(t)\right\| \leqslant C h^{r-1}\left[\left\|u^{0}\right\|_{r+1}+\left\|u_{t}^{0}\right\|_{r}\right]
$$

3. $L^{\infty}$-Estimates for Fully Discrete Approximations. The results of the previous section although mathematically interesting in their own right, will basically be used as a tool in obtaining the $L^{\infty}(\Omega)$-estimates for the fully discrete approximations, which are the objectively important schemes from a practical standpoint.

We first recall some notation from [2]. $T_{h}$ restricted to $S_{h}$ possesses a set of purely imaginary eigenvalues $\left\{\eta_{ \pm j}\right\}_{j=1}^{M}$ given by

$$
\eta_{ \pm j}= \pm i\left(\mu_{j}^{h}\right)^{1 / 2}
$$

and a corresponding set of eigenfunctions

$$
\Phi_{ \pm j}=\frac{1}{\sqrt{2}}\left(\begin{array}{c}
\psi_{j}^{h} \\
\pm i\left(\mu_{j}^{h}\right)^{-1 / 2} \psi_{j}^{h}
\end{array}\right), \quad j=1,2, \ldots, M .
$$

The set $\left\{\Phi_{ \pm j}\right\}_{j=1}^{M}$ is orthonormal with respect to the inner product $((\cdot, \cdot))_{h}$, extended to the complex case by $((\Phi, \Psi))_{h}=\left(\varphi_{1}, \bar{\psi}_{1}\right)+\left(T_{h} \varphi_{2}, \bar{\psi}_{2}\right)$, for

$$
\Phi=\left(\begin{array}{c}
\varphi_{1} \\
\varphi_{2}
\end{array}\right), \quad \Psi=\left(\begin{array}{l}
\psi_{1} \\
\psi_{2}
\end{array}\right) \in S_{h},
$$

where $\bar{z}$ denotes the complex conjugate of $z$. We see that the assumption (1.27) becomes

$$
\sum_{j}\left|\eta_{j}\right|^{2 J_{0}} \leqslant C<\infty
$$


We first present a preliminary lemma on an estimate in the maximum norm of the eigenvectors $\psi_{j}^{h}$ of $T_{h}$, the proof of which follows from [3, Lemma 3.1]. For convenience in notation we set $\psi_{j}^{h}=\psi_{-j}^{h}$.

Lемма 3.1. With notation as above, there exists a $K$ depending only on $N$ and a constant $C$ for which

$$
\left|\psi_{j}^{h}\right| \leqslant C\left|\eta_{j}\right|^{-2 K} \quad \text { for all } j
$$

in fact, $K=[N / 2]+1$.

We point out that Lemma 3.1 is a slight refinement of Lemma 3.1 of [3] expressly for our present purposes. The details of the proof are the same, except that we exploit the fact that the iterative argument used may be carried out in $[N / 2]+1$ steps, which gives the convenient value $K=[N / 2]+1$. We have prior to this also exploited this fact in Lemma 2.6, where we use the same iterative argument.

We shall need to work with functions of the operator $L_{h}$. Let $f$ be a function analytic in a neighborhood of the spectrum $\left\{\eta_{ \pm j}^{-1}\right\}_{j=1}^{M}$ of $L_{h}$ on $S_{h}$. Then, if $X \in$ $\mathbf{L}^{2}$, we have the spectral representation,

$$
f\left(L_{h}\right) X=\sum_{j} f\left(\eta_{j}^{-1}\right)\left(\left(X, \Phi_{j}\right)\right)_{h} \Phi_{j} .
$$

We shall, in the first instance, restrict our attention to fully discrete schemes associated with rational functions $r$ of class i-II, defined in Section 1. This is no loss in generality since our whole analysis will go through for (conditionally stable) schemes associated with rational functions of class $\mathrm{i}-\mathrm{I}$, under a restriction on $k / h$, provided (1.18) holds; cf. e.g., [2, Lemma 3.3].

With $r(z)$ as in Section 1 we define for $n=0,1,2, \ldots$,

$$
F_{n}(z)=r^{n}(z)-e^{-n z}
$$

Then, if $\left\{W^{n}\right\}_{n \geqslant 0}$ is the solution of (1.26) and $V$ is the solution of (1.15), we have

$$
W^{n}-V(n k)=F_{n}\left(k L_{h}\right) V(0)
$$

We first present a preliminary stability result.

LEMMA 3.2. Let $r(z)$ be a rational function of class i-II. Let $S$ be an integer such that $S \geqslant J_{0}+[N / 2]+1$, where $J_{0}$ is defined by (1.27) or (3.3). Then, there exists a constant $C=C\left(t^{*}\right)$ such that for $X \in \mathrm{L}^{2}$,

$$
\left|\left(F_{n}\left(k L_{h}\right) T_{h}^{2 S+l} X\right)_{1}\right| \leqslant C k^{l-1}\|X X\|_{h}, \quad 1 \leqslant l \leqslant \nu+1, n=0,1, \ldots,\left[t^{*} / k\right],
$$

and also,

$$
\left|\left(F_{n}\left(k L_{h}\right) T_{h}^{2 S} X\right)_{1}\right| \leqslant C\left|\|X \mid\|_{h}, \quad n=0,1,2, \ldots,\left[t^{*} / k\right]\right.
$$

If in addition (1.18) is satisfied, then we also have 
and

$$
\begin{aligned}
\left|\left(F_{n}\left(k L_{h}\right) T_{h}^{2 S+l} X\right)_{2}\right| \leqslant C k^{l-1} h^{-1}\|X\|_{h}, & \\
1 & \leqslant l \leqslant \nu+1, n=0,1, \ldots,\left[t^{*} / k\right],
\end{aligned}
$$

$$
\left|\left(F_{n}\left(k L_{h}\right) T_{h}^{2 S} X\right)_{2}\right| \leqslant C h^{-1}\|X \mid\|_{h}, \quad n=0,1, \ldots,\left[t^{*} / k\right] .
$$

Proof. We have

$$
\left(F_{n}\left(k L_{h}\right) T_{h}^{2 S+l} X\right)_{1}=\sum_{j} F_{n}\left(k \eta_{j}^{-1}\right) \eta_{j}^{2 S+l}\left(\left(X, \Phi_{j}\right)\right)_{h} \Phi_{j, 1} .
$$

Hence, by (3.2)

$$
\left|\left(F_{n}\left(k L_{h}\right) T_{h}^{2 S+l} X\right)_{1}\right| \leqslant 2^{-1 / 2}\left(\sum_{j}\left|F_{n}\left(k \eta_{j}^{-1}\right)\right|\left|\eta_{j}\right|^{2 S+l}\left|\psi_{j}^{n}\right|\right)\|X\|_{h} .
$$

Now, using [2, Lemma 3.1], we conclude that for $n=0,1,2, \ldots$,

$$
\left|F_{n}\left(k \eta_{j}^{-1}\right)\right| \leqslant C n k^{l}\left|\eta_{j}\right|^{-l} \leqslant C k^{l-1}\left|\eta_{j}\right|^{-l}, \quad 1 \leqslant l \leqslant \nu+1 .
$$

Hence, for $K=[N / 2]+1$, using (3.4), we conclude that

$$
\begin{aligned}
\left|\left(F_{n}\left(k L_{h}\right) T_{h}^{2 S+l} X\right)_{1}\right| & \leqslant C k^{l-1}\left(\sum_{j}\left|\eta_{j}\right|^{2 S-2 K}\right)\|X\|_{h} \\
& \leqslant C k^{l-1}\left(\sum_{j}\left|\eta_{j}\right|^{\left(2 S-2 K-2 J_{0}\right)+2 J_{0}}\right)\|X\|_{h} \\
& \leqslant C k^{l-1}\|X \mid\|_{h},
\end{aligned}
$$

where the last inequality follows by the hypothesis on $S$ and (3.3). To prove (3.8) we note that, by the definition of $F_{n}$, since the $\eta_{j}$ are purely imaginary:

$$
\left|F_{n}\left(k \eta_{j}^{-1}\right)\right| \leqslant 2 \text { all } j, n \text {. }
$$

The proof then follows from similar calculations as above.

Now (3.2) gives,

$$
\left|\left(F_{n}\left(k L_{h}\right) T_{h}^{2 S+l} X\right)_{2}\right| \leqslant 2^{-1 / 2}\left(\sum_{j}\left|F_{n}\left(k \eta_{j}^{-1}\right)\right|\left|\eta_{j}\right|^{2 S+l-1}\left|\psi_{j}^{h}\right|\right)\|X \mid\|_{h} .
$$

By (1.18) we conclude that $\left|\eta_{j}\right|^{-1} \leqslant C h^{-1}$ for all $j$; and the remainder of the proof of (3.9) and (3.10) follows, as that for (3.7) and (3.8) above.

We now find maximum norm estimates for the components of $W^{n}-V(n k)$ with initial conditions of the type (1.16). For the proofs we need to define some auxiliary functions. Given $k>0$, let $Q$ be the least integer for which

$$
k \lambda_{j}^{1 / 2}>1 \text { for } j \geqslant Q
$$

Then, given $v \in L^{2}$, we define

$$
v^{(k)}=\sum_{j=1}^{Q-1}\left(v, \varphi_{j}\right) \varphi_{j}
$$


It is easily seen that $v^{(k)} \in C^{\infty}(\Omega)$ and that it satisfies

$$
\left\|v^{(k)}\right\|_{S+m} \leqslant k^{-m}\|v\|_{S} \text { for all } m \geqslant 0 \text { and real } S,
$$

and

$$
\left\|v-v^{(k)}\right\|_{p} \leqslant k^{S-p}\|v\|_{S} \text { for all } S \geqslant 0 \text { and all real } p \leqslant S
$$

We define

$$
U^{0(k)}=\left(\begin{array}{l}
u^{0(k)} \\
u_{t}^{0(k)}
\end{array}\right)
$$

LEMMA 3.3. Let $r(z)$ and the integer $S$ satisfy the hypotheses of Lemma 3.2, and let the initial condition in (1.15) and (1.26) be given by $W^{0}=V(0)=$ $T_{h}^{2 S} L^{2 S} U^{0}$. Then, for some constant $C=C\left(t^{*}\right)$ we have

$$
\begin{aligned}
& \max _{0 \leqslant n<\left[t^{*} / k\right]}\left|W_{1}^{n}-V_{1}(n k)\right| \\
& \quad \leqslant C\left\{h^{r}\left(\left\|u^{0}\right\|_{2 s+r+1}+\left\|u_{t}^{0}\right\|_{2 s+r}\right)+k^{\nu}\left(\left\|u^{0}\right\|_{2 s+\nu+1}+\left\|u_{t}^{0}\right\|_{2 s+\nu}\right)\right\} .
\end{aligned}
$$

If in addition $T_{h}$ satisfies (1.18) and $k / h \leqslant C$ for any $C<\infty$, we also have

$$
\begin{aligned}
& \max _{0 \leqslant n<\left[t^{*} / k\right]}\left|W_{2}^{n}-V_{2}(n k)\right| \\
& \quad \leqslant C\left\{k^{\nu-1}\left(\left\|u^{0}\right\|_{2 S+\nu+1}+\left\|u_{t}^{0}\right\|_{2 S+\nu}\right)+h^{r-1}\left(\left\|u^{0}\right\|_{2 S+r+1}+\left\|u_{t}^{0}\right\|_{2 S+r}\right)\right\} .
\end{aligned}
$$

Proof. Following [2] we can write, by (3.6), using our hypothesis for $V(0)=W^{0}$,

$$
\begin{aligned}
W^{n}-V(n k)= & F_{n}\left(k L_{h}\right) T_{h}^{2 S} L^{2 S} U^{0}=F_{n}\left(k L_{h}\right) T_{h}^{2 S} L^{2 S}\left(U^{0}-U^{0(k)}\right) \\
& +\sum_{l=0}^{\nu} F_{n}\left(k L_{h}\right) T_{h}^{l+2 S}\left(T-T_{h}\right) L^{l+1+2 S} U^{0(k)} \\
& +F_{n}\left(k L_{h}\right) T_{h}^{\nu+1+2 S} L^{\nu+1+2 S} U^{0(k)}
\end{aligned}
$$

from which, by Lemma 3.2,

$$
\begin{gathered}
\left|W_{1}^{n}-V_{1}(n k)\right| \leqslant C\left(\left\|L^{2 S}\left(U^{0}-U^{0(k)}\right)\right\|_{h}+\left\|K\left(T-T_{h}\right) L^{2 S+1} U^{0(k)}\right\|_{h}\right. \\
+\sum_{l=1}^{\nu} k^{l-1}\left\|\left(T-T_{h}\right) L^{l+1}+2 S U^{0(k)}\right\|_{h} \\
\left.+k^{\nu}\left\|L^{\nu+1+2 S} U^{0(k)}\right\|_{h}\right) .
\end{gathered}
$$

For the first term on the right-hand side of the above inequality, using (1.8), (3.11), 
(3.12), we have

$$
\begin{aligned}
&\left\|L^{2 S}\left(U^{0}-U^{0(k)}\right)\right\|_{h}^{2} \\
&=\left\|L^{S}\left(u^{0}-u^{0(k)}\right)\right\|^{2}+\left(T_{h} L^{S}\left(u_{t}^{0}-u_{t}^{0(k)}\right), L^{S}\left(u_{t}^{0}-u_{t}^{0(k)}\right)\right) \\
& \leqslant k^{2 \nu}\left\|u^{0}\right\|_{2 S+\nu}^{2}+\left(\left(T_{h}-T\right) L^{S}\left(u_{t}^{0}-u_{t}^{0(k)}\right), L^{S}\left(u_{t}^{0}-u_{t}^{0(k)}\right)\right) \\
&+\left(T L^{S}\left(u_{t}^{0}-u_{t}^{0(k)}\right), L^{S}\left(u_{t}^{0}-u_{t}^{0(k)}\right)\right) \\
& \leqslant k^{2 v}\left\|u^{0}\right\|_{2 S+\nu}^{2}+\left\|\left(T_{h}-T\right) L^{S}\left(u_{t}^{0}-u_{t}^{0(k)}\right)\right\|\left\|L^{S}\left(u_{t}^{0}-u_{t}^{0(k)}\right)\right\| \\
&+\left\|T L^{S}\left(u_{t}^{0}-u_{t}^{0(k)}\right)\right\|_{1}\left\|L^{S}\left(u_{t}^{0}-u_{t}^{0(k)}\right)\right\|_{-1} \\
& \leqslant k^{2 \nu}\left\|u^{0}\right\|_{2 S+\nu}^{2}+C h^{r}\left\|u_{t}^{0}\right\|_{2 S+r-2} k^{\nu}\left\|u_{t}^{0}\right\|_{2 S+\nu}+k^{2 \nu}\left\|u_{t}^{0}\right\|_{2 S+\nu-1}^{2} \\
& \leqslant C\left\{h^{r}\left\|u_{t}^{0}\right\|_{2 S+r-2}+k^{\nu}\left(\left\|u^{0}\right\|_{2 S+\nu}+\left\|u_{t}^{0}\right\|_{2 S+\nu}\right)\right\}^{2}
\end{aligned}
$$

We estimate the second term in the right-hand side of (3.17), using (1.8) and (3.11), by

$$
\text { (3.19) }\left\|\left(T-T_{h}\right) L^{2 S+1} U^{0(k)}\right\|_{h}=\left\|\left(T-T_{h}\right) L^{S+1} u^{0(k)}\right\| \leqslant C h^{r}\left\|u^{0}\right\|_{2 S+r} \text {. }
$$

For the third term we remark that for $l+1$ even

$$
L^{l+1+2 S}=(-1)^{(l+1) / 2+S}\left(\begin{array}{cc}
L^{(l+1) / 2+S} & 0 \\
0 & L^{(l+1) / 2+S}
\end{array}\right)
$$

and for $l+1$ odd

$$
L^{l+1+2 S}=(-1)^{l / 2+S}\left(\begin{array}{cc}
0 & -L^{l / 2+S} \\
L^{l / 2+1+S} & 0
\end{array}\right) .
$$

Hence,

$$
\begin{aligned}
\left\|\left(T-T_{h}\right) L^{l+1+2 S} U^{O(k)}\right\|_{h} \\
\leqslant \begin{cases}\left\|\left(T-T_{h}\right) L^{S+(l+1) / 2} u_{t}^{0(k)}\right\|, & l+1 \text { even } \\
\left\|\left(T-T_{h}\right) L^{S+1+l / 2} u^{0(k)}\right\|, & l+1 \text { odd }\end{cases}
\end{aligned}
$$

Then, by (1.8) and (3.11) we see that

(3.20) $\sum_{l=1}^{\nu} k^{l-1}\left\|\left(T-T_{h}\right) L^{l+1+2 S} U^{0(k)}\right\|_{h} \leqslant C h^{r}\left(\left\|u_{t}^{0}\right\|_{2 S+r}+\left\|u^{0}\right\|_{2 S+r+1}\right)$.

Finally, for the last term of (3.17), if $\nu+1$ is even, we obtain, using (1.8), (3.11), (3.12), that 


$$
\begin{aligned}
& k^{2 v}\left\|L^{\nu+1+2 S} U^{0(k)}\right\|_{h}^{2} \\
& =k^{2 \nu}\left\{\left\|L^{S+(\nu+1) / 2} u^{0(k)}\right\|^{2}+\left(T_{h} L^{S+(\nu+1) / 2} u_{t}^{0(k)}, L^{S+(\nu+1) / 2} u_{t}^{0(k)}\right)\right\} \\
& =k^{2 \nu}\left\{\left\|u^{0(k)}\right\|_{2 S+\nu+1}^{2}+\left(\left(T_{h}-T\right) L^{S+(\nu+1) / 2} u_{t}^{0(k)}, L^{S+(\nu+1) / 2} u_{t}^{0(k)}\right)\right. \\
& \left.+\left(T L^{S+(\nu+1) / 2} u_{t}^{0(k)}, L^{S+(\nu+1) / 2} u_{t}^{0(k)}\right)\right\} \\
& \leqslant C k^{2 \nu}\left\{\left\|u^{0}\right\|_{2 S+\nu+1}^{2}+h^{r}\left\|u_{t}^{0(k)}\right\|_{2 S+r+\nu-1}\left\|u_{t}^{0(k)}\right\|_{2 S+\nu+1}\right. \\
& \left.+\left\|u_{t}^{0(k)}\right\|_{2 S+v}^{2}\right\} \\
& \leqslant C\left\{k^{2 \nu}\left\|u^{0}\right\|_{2 S+\nu+1}^{2}+h^{r} k^{\nu}\left\|u_{t}^{0}\right\|_{2 S+r}\left\|u_{t}^{0}\right\|_{2 S+\nu}+k^{2 \nu}\left\|u_{t}^{0}\right\|_{2 S+\nu}^{2}\right\} \\
& \leqslant C\left\{k^{\nu}\left(\left\|u^{0}\right\|_{2 S+\nu+1}+\left\|u_{t}^{0}\right\|_{2 S+\nu}\right)+h^{r}\left\|u_{t}^{0}\right\|_{2 S+r}\right\}^{2} \text {. }
\end{aligned}
$$

Similarly, for $\nu+1$ odd we obtain

$$
\begin{aligned}
& k^{2 \nu}\left\|L^{\nu+1}+2 S U^{0(k)}\right\|_{h}^{2} \\
& \quad \leqslant C\left\{k^{\nu}\left(\left\|u^{0}\right\|_{2 S+\nu+1}+\left\|u_{t}^{0}\right\|_{2 S+\nu}\right)+h^{r}\left\|u^{0}\right\|_{2 S+r+1}\right\}^{2} .
\end{aligned}
$$

(3.14) now follows from (3.17)-(3.21').

We now note, using (3.16), (3.9) and (3.10) that $\left|W_{2}^{n}-V_{2}(n k)\right|$ is bounded by a factor of $\mathrm{Ch}^{-1}$ times the right-hand side of (3.17). Hence, (3.15) follows immediately.

We now state the first main result of this section, the proof of which follows from Theorem 2.1 and Lemma 3.3.

THEOREM 3.1. Let $r(z)$ and the integer $S$ satisfy the hypotheses of Lemma 3.2. Let $S_{0}$ be defined as in Theorem 2.1, and let $V(0)=T_{h}^{2 S} L^{2 S} U^{0}$. Then, for some constant $C=C\left(t^{*}\right)$ we have that

$$
\max _{0 \leqslant n \leqslant\left[t^{*} / k\right]}\left|W_{1}^{n}-u(n k)\right|
$$

$$
\begin{aligned}
\leqslant C\left\{\gamma(h)\left(\left\|u^{0}\right\|_{S_{0}}+\left\|u_{t}^{0}\right\|_{s_{0}-1}\right)+\right. & h^{r}\left(\left\|u^{0}\right\|_{2 S+r+1}+\left\|u_{t}^{0}\right\|_{2 S+r}\right) \\
& \left.+k^{\nu}\left(\left\|u^{0}\right\|_{2 S+\nu+1}+\left\|u_{t}^{0}\right\|_{2 S+\nu}\right)\right\} .
\end{aligned}
$$

If in addition (1.18) is satisfied, and $k / h \leqslant C$ for any $C<\infty$, then

$$
\max _{0<n<\left[t^{*} / k\right]}\left|W_{2}^{n}-D_{t} u(n k)\right|
$$

$$
\begin{aligned}
\leqslant C\left\{\gamma(h)\left(\left\|u^{0}\right\|_{S_{0}+1}+\left\|u_{t}^{0}\right\|_{S_{0}}\right)\right. & +h^{r-1}\left(\left\|u^{0}\right\|_{2 S+r+1}+\left\|u_{t}^{0}\right\|_{2 S+r}\right) \\
& \left.+k^{\nu-1}\left(\left\|u^{0}\right\|_{2 S+\nu+1}+\left\|u_{t}^{0}\right\|_{2 S+\nu}\right)\right\} .
\end{aligned}
$$

We now analyze the error in the fully discrete approximation obtained with initial condition of the type (1.20). As in the semidiscrete case, we need no additional conditions for the estimate of the error in the second component. We first need a preliminary stability lemma. 
LEMMA 3.4. Let $r(z)$ and the integer $S$ satisfy the hypotheses of Lemma 3.2.

Then there is a constant $C=C\left(t^{*}\right)$ such that for $X \in \mathrm{L}^{2}$ :

$$
\begin{array}{r}
\left|\left(F_{n}\left(k L_{h}\right) T_{h}^{2 S+l+1} X\right)_{i}\right| \leqslant C k^{l-1}\left|\|X \mid\|_{h}, \quad i=1,2,1 \leqslant l \leqslant \nu+1,\right. \\
n=0,1,2, \ldots,\left[t^{*} / k\right],
\end{array}
$$

and

$$
\left|\left(F_{n}\left(k L_{h}\right) T_{h}^{2 S+1} X\right)_{i}\right| \leqslant C\left|\|X \mid\|_{h}, \quad i=1,2, n=0,1, \ldots,\left[t^{*} / k\right] .\right.
$$

Proof. As in the proof of Lemma 3.2, we have for $i=1,2$,

$$
\begin{aligned}
\left|\left(F_{n}\left(k L_{h}\right) T_{h}^{2 S+l+1} X\right)_{i}\right| & \leqslant C\left(\sum_{j}\left|F_{n}\left(k \eta_{j}^{-1}\right) \| \eta_{j}\right|^{2 S+l+2-i}\left|\psi_{j}^{h}\right|\right)\|X \mid\|_{h} \\
& \leqslant C k^{l-1}\left(\sum_{j}\left|\eta_{j}\right|^{\left(2 S+2-i-2 K-2 J_{0}\right)+2 J_{0}}\right)\|X \mid\|_{h} .
\end{aligned}
$$

Hence, the condition $S \geqslant K+J_{0}$ is sufficient to establish (3.24). The proof of (3.25) also follows easily.

The analog to Lemma 3.3 is now

LEMMA 3.5. Let $r(z)$ and $S$ satisfy the hypotheses of Lemma 3.2 and $W^{0}=$ $V(0)=T_{h}^{2 S+1} L^{2 S+1} U^{0}$. Then, for some constant $C=C\left(t^{*}\right)$ we have for $i=1,2$,

$$
\begin{aligned}
\max _{0 \leqslant n \leqslant\left[t^{*} / k\right]}\left|W_{i}^{n}-V_{i}(n k)\right| \leqslant C\left\{h^{r}\left(\left\|u^{0}\right\|_{2 S+r+2}+\left\|u_{t}^{0}\right\|_{2 S+r+1}\right)\right. \\
\\
\left.+k^{\nu}\left(\left\|u^{0}\right\|_{2 S+\nu+2}+\left\|u_{t}^{0}\right\|_{2 S+\nu+1}\right)\right\} .
\end{aligned}
$$

Proof. We have

$$
\begin{aligned}
W^{n}-V(n k)= & F_{n}\left(k L_{h}\right) T_{h}^{2 S+1} L^{2 S+1} U^{0} \\
= & F_{n}\left(k L_{h}\right) T_{h}^{2 S+1} L^{2 S+1}\left(U^{0}-U^{0(k)}\right) \\
& +\sum_{l=0}^{\nu} F_{n}\left(k L_{h}\right) T_{h}^{2 S+l+1}\left(T-T_{h}\right) L^{2 S+l+2} U^{0(k)} \\
& +F_{n}\left(k L_{h}\right) T_{h}^{2 S+\nu+2} L^{2 S+\nu+2} U^{0(k)},
\end{aligned}
$$

from which, by Lemma 3.4 , it follows that

$$
\begin{gathered}
\left|W_{i}^{n}-V_{i}(n k)\right| \leqslant C\left(\left\|L^{2 S+1}\left(U^{0}-U^{0(k)}\right)\right\|_{h}+\left\|\left(T-T_{h}\right) L^{2 S+2} U^{0(k)}\right\|_{h}\right. \\
\quad+\sum_{l=1}^{\nu} k^{l-1}\left\|\left(T-T_{h}\right) L^{l+2 S+2} U^{0(k)}\right\|_{h} \\
\left.+k^{\nu}\left\|L^{\nu+2 S+2} U^{0(k)}\right\|_{h}\right)
\end{gathered}
$$

and (3.26) follows by using entirely similar techniques of estimation with the ones of Lemma 3.3. 
Combining the results of Theorem 2.2 and Lemma 3.5 , we obtain

THEOREM 3.2. Let $r$ and the integer $S$ satisfy the hypotheses of Lemma 3.2, and let $S_{0}$ be defined as in Theorem 2.1 and let $V(0)=T_{h}^{2 S+1} L^{2 S+1} U^{0}$. Then, for some constant $C=C\left(t^{*}\right)$ :

$$
\begin{aligned}
\max _{0 \leqslant n \leqslant\left[t^{*} / k\right]}\left|W_{1}^{n}-u(n k)\right| & \\
\leqslant C\left\{\gamma(h)\left(\left\|u^{0}\right\|_{S_{0}}+\left\|u_{t}^{0}\right\|_{S_{0}-1}\right)+\right. & h^{r}\left(\left\|u^{0}\right\|_{2 S+r+2}+\left\|u_{t}^{0}\right\|_{2 S+r+1}\right) \\
& \left.+k^{\nu}\left(\left\|u^{0}\right\|_{2 S+\nu+2}+\left\|u_{t}^{0}\right\|_{2 S+\nu+1}\right)\right\}
\end{aligned}
$$

and

$$
\begin{aligned}
\max _{0 \leqslant n \leqslant\left[t^{*} \mid k\right]}\left|W_{2}^{n}-D_{t} u(n k)\right| & \\
\leqslant C\left\{\gamma(h)\left(\left\|u^{0}\right\|_{S_{0}+1}+\left\|u_{t}^{0}\right\|_{S_{0}}\right)\right. & +h^{r}\left(\left\|u^{0}\right\|_{2 S+r+2}+\left\|u_{t}^{0}\right\|_{2 S+r+1}\right) \\
& \left.+k^{\nu}\left(\left\|u^{0}\right\|_{2 S+\nu+2}+\left\|u_{t}^{0}\right\|_{2 s+\nu+1}\right)\right\} .
\end{aligned}
$$

We now turn to convergence results when rational functions of class i-I are used. Since the proofs of the results are entirely analogous to those for class i-II, we shall merely point out the major technical changes, and conclude by stating the results.

THEOREM 3.3. Let $r$ be of class i-I and suppose that condition (1.18) holds. Then, there exists a constant $\beta=\beta(\alpha)$, where $\alpha$ is as in Definition I, such that if $k / h \leqslant$ $\beta$, the following estimates hold.

(a) If $S \geqslant[N / 2]+J_{0}+1$, and $W^{0}=V(0)=T_{h}^{2 S} L^{2 S} U^{0}$, then the estimates (3.22) and (3.23) hold.

(b) If $W^{0}=T_{h}^{2 S+1} L^{2 S+1} U^{0}$, with $S \geqslant[N / 2]+J_{0}+1$, then the estimates (3.27) and (3.28) hold.

Proof. From [2, Lemma 3.1], if $r$ is of class i-I, the inequality (3.10a) holds for $j$ such that $k\left|\eta_{j}\right|^{-1} \leqslant \alpha$. Thus, under the condition (1.18), if $\beta(\alpha)=\alpha C^{1 / 2}$, with $C$ the constant of (1.18), and $k h^{-1} \leqslant \beta$, then, for all $j, k\left|\eta_{j}\right|^{-1} \leqslant C^{-1 / 2} k h^{-1} \leqslant \alpha$. Hence, (3.10a) holds for all $j$. The remainder of the proofs of (a) and (b) are the same as for the corresponding results of Theorems 3.1 and 3.2 , respectively.

4. Remarks. The convergence results of Section 3 rely implicitly on the asymptotic distribution of the eigenvalues of the elliptic operator $L$ on $\Omega$. More specifically, our results assert that for optimal convergence in $L^{\infty}(\Omega)$ of the error, it is sufficient to choose $W^{0}=T_{h}^{2 S} L^{2 S} U^{0}$ with $S=[N / 2]+J_{0}+1$ (Theorem 3.1), where $J_{0}$ is any integer satisfying (1.27). Thus, for actual implementation, one must find a convenient $J_{0}$. This we do in the present setting as follows:

It is known from the work of Browder [5], among others, that under the conditions we have imposed on $\Omega$ and $L$,

$$
\lim _{j \rightarrow \infty} j / \lambda_{j}^{N / 2}=\omega_{N} \int_{\Omega}\left[\operatorname{DET}\left(a_{i j}(x)\right)\right]^{1 / 2} d x,
$$


where

$$
\omega_{N}=\left[(2 \sqrt{\pi})^{N} \Gamma(1+N / 2)\right]^{-1}
$$

It is also well known that in the canonical example of the standard Galerkin method (1.12) there is a rearrangement of the indices $j$ such that

$$
\mu_{j}^{h} \leqslant \lambda_{j}^{-1}
$$

From (4.1), (1.27) and (4.2), a simple computation shows that we may choose

$$
J_{0}=J_{0}(\Omega)=N .
$$

Thus, for optimal $L^{\infty}(\Omega)$-convergence of the error, it suffices to choose

$$
W^{0}=T_{h}^{2 S} L^{2 S} U^{0}=\left(\begin{array}{c}
T_{h}^{S} L^{S} u^{0} \\
T_{h}^{S} L^{S} u_{t}^{0}
\end{array}\right),
$$

with $S=[N / 2]+N+1$. As pointed out before, the choice (4.3) is accomplished with the solution of

$$
\begin{cases}3 N+2, & N \text { even, } \\ 3 N+1, & N \text { odd },\end{cases}
$$

linear systems of equations initially, using the same real matrix.

For a survey of results of the type (4.1) we refer to [6].

Division of Applied Sciences

Harvard University

Cambridge, Massachusetts 02138

Department of Mathematics

University of Tennessee

Knoxville, Tennessee 37916

1. G. A. BAKER, "Error estimates for finite element methods for second order hyperbolic equations," SIAM J. Numer. Anal., v. 13, 1976, pp. 564-576.

2. G. A. BAKER \& J. H. BRAMBLE, Semidiscrete and Single Step Fully Discrete Approximations for Second Order Hyperbolic Equations, Rep. No. 22, Centre de Mathématiques Apliquées, Ecole Polytechnique, Paris, 1977. Also RAIRO Analyse Numérique, v. 13, 1979, pp. 75-100.

3. G. A. BAKER, J. H. BRAMBLE \& V. THOMÉE, "Single step Galerkin approximations for parabolic problems," Math. Comp., v. 31, 1977, pp. 818-847.

4. J. H. BRAMBLE, A. H. SCHATZ, V. THOMÉE \& L. B. WAHLBIN, "Some convergence estimates for semidiscrete Galerkin type approximations for parabolic equations," SIAM J. Numer. Anal, v. 14, 1977, pp. 218-241.

5. F. E. BROWDER, "Asymptotic distribution of eigenvalues and eigenfunctions for nonlocal elliptic boundary value problems. I," Amer. J. Math., v. 87, 1965, pp. 175-195.

6. C. CLARK, "The asymptotic distribution of eigenvalues and eigenfunctions for elliptic boundary value problems," SIAM Rev., v. 9, 1967, pp. 627-646.

7. M. CROUZEIX, Sur l'Approximation des Équations Différentielles Opérationelles Linéaires par des Méthodes de Runge-Kutta, Thèse, Université Paris-VI, Paris, 1975.

8. T. DUPONT, " $L 2$-estimates for Galerkin methods for second order hyperbolic equations," SIAM J. Numer. Anal., v. 10, 1973, pp. 880-889.

9. J. FREHSE \& R. RANNACHER, “Asymptotic $L^{\infty}$-error estimates for linear finite element approximations of quasilinear boundary value problems," SIAM J. Numer. Anal., v. 15, 1978, pp. 418-431. 
10. F. NATTERER, “Über die punktweise Konvergenz finiter Elemente,” Numer. Math., v. 25 , 1975, pp. 67-77.

11. J. A. NITSCHE, $L^{\infty}$-Convergence for Finite Element Approximation, 2 nd Conf. on Finite Elements, Rennes, France, May 12-14, 1975.

12. J. A. NITSCHE, "On $L^{\infty}$-convergence of finite element approximations to the solution of a nonlinear boundary value problem," Topics in Numerical Analysis III, Proc. Roy. Irish Acad. Conference on Numerical Analysis, 1976 (J. J. H. Miller, Ed.), Academic Press, London, 1977, pp. 317-325.

13. R. RANNACHER, "Zur $L^{\infty}$-Konvergenz linearer finiter Elemente beim Dirichlet-problem," Math. Z., ,v. 149, 1976, pp. 69-77.

14. R. SCOTT, "Optimal $L^{\infty}$-estimates for the finite element method on irregular meshes," Math. Comp., v. 30, 1976, pp. 681-697.

15. L. B. WAHLBIN, "On maximum norm error estimates for Galerkin approximations to one-dimensional second order parabolic boundary value problems," SIAM J. Numer. Anal., v. 12, 1975, pp. 177-187.

16. M. F. WHEELER, " $L$-estimates of optimal orders for Galerkin methods for one-dimensional second order parabolic and hyperbolic equations," SIAM J. Numer. Anal., v. 10, 1973, pp. 908-913. 